\title{
Sustainable development and heritage: "trabocchi" and the rules for building on the coast
}

\author{
M. C. Forlani, M. Borrone \& D. Radogna \\ DiTAC-Department of Technology for Build Environment, \\ Faculty of Architecture, University "G. D'Annunzio" - Pescara-Chieti, \\ Italy
}

\begin{abstract}
The purpose of this study is to promote tourism as a basis for the sustainable development of the territory, in particular of the Teatina coast in the province of Chieti - Abruzzi. This study is focused on the maintenance and recovery of the "trabocchi" (ancient wooden fishing huts built on stilts) which are paradigmatic instances of the building praxis of the "genius loci". The study is aimed at pointing out the role played by these constructions in determining conditions for sustainable development in relation to bathing facilities. We must consider that the elements on which tourist promotion of the territory is based can be easily put aside in a project which is not understood in all its implications and aspects. In these highly vulnerable areas, tourism has an impact on both physical and image development and might be relevant in causing strong anthropic pressure on the natural environment. The improper use of the territory can quickly cancel cultural memory and threaten one's sense of history, giving space to a landscape which is homologated or even alienated from the false icons of local material culture. The study we present here is the result of a commission of the Province of Chieti to carry out an integrated project where each action (exploitation of nature, bathing facilities, link with the urban and inland agricultural, recovery and re-use of emerging manufactured products for services, sustainable alternate mobility) is strongly connected to the others, and each choice has to be valued according to the results in the other sectors.

The first part of this work involves formulating rules to build new "trabocchi" and determine the elements needed to define the required surroundings. In short, these new constructions must be located within acceptable "scenery" that reflect
\end{abstract}


their traditional types of natural location, and therefore provide the basis of a new pattern of tourism and the individuation of sustainable indicators to set compatible facilities on the above mentioned coast.

Keywords: sustainable development, coastal environment, trabocchi, heritage.

\section{Introduction}

Starting from the recovery of a typical structure constituted by original, manufactured products, the aim of delineating the parameters that must be set for the sustainable development of a specific territory necessitates a brief report on these products, as well as a critical overview of the state of the art. The trabocchi are constructions for inshore fishing which could have taken origin in the XIII century, surely attested in the XVII century; they represent a demonstration of genius loci which make full use of local material resources and encouraged the "transfer" of technologies in an appropriate way; this is the reason why they will be considered a useful instance of "sustainable practice" for the building of manufactured products on the sea. The Teatine coast is characterized by the presence of a remarkable number of trabocchi. A structural analysis of them shows the elements which compose the system (footbridge, flat of fishing, stabilizing grid, fishing device, functional equipment); furthermore, it is possible to recognize the evolutionary process which constitutes one of the characteristics of such manufactured products. One especially refers to the changes due to the transfer of technologies (for instance the one that happened in concomitance with the railway building) and the modifications due to the change of use, now acting, ranging from fishing to "service" of the bathing tourism system; in the first case, mutations prevalently regarded the technical-constructive device, in the second case they take into account the dimensional morphological system for reasons connected to the possible widened fruition and, therefore, to the necessary security.

Until the 1970s, the interventions of maintenance, made by the same men working on the trabocchi, did not determine, on the whole, relevant changes in the system arrangement. The documentation of those years still shows the trabocchi as "emanations" of a place sufficiently uncontaminated from strong urbanization. Their anthropological context was mainly limited to rare

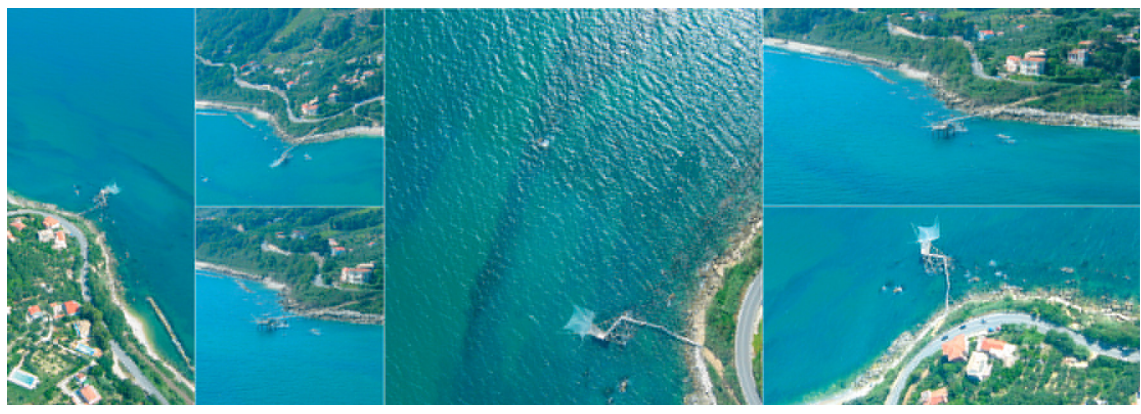

Figure 1: Images of the trabocchi along the Teatine coast. 
settlements, little villages and isolated villas which progressively had been integrated into a natural and agricultural landscape without producing a break with tradition; the railway itself had been "overwhelmed" by the surrounding landscape, thanks also to some literary narrations (See G. D'Annunzio, Trionfo della morte, 1894) which also contributed to a representation of the railway as a "character" of the landscape itself. Conversely, in the following years the socioeconomic evolution has showed its negative aspects also in this territory: a sudden urban growth was being achieved without considering any environmental consequences; the choice of technologies and building materials extraneous to the local culture; the progressive shift to a mass tourism which is unlikely to be supported by such a "fragile" natural environment as the territory above mentioned, in terms of the configuration of its landscape, as well as of its geological consistency. It is, in particular, little more than 20 kilometres of high coast with high hydro-geological risk for a particular vulnerable cliff. During this time of rapid growth and transformation, the trabocchi were prevalently abandoned, the people leaving to seek more profitable work.

In more recent times a politics based on specific local interventions has allowed a recovery process of this characteristic architectonic-cultural inheritance so as to induce various territorial corporations to develop appropriate projects; the Abruzzi area, in particular, enacted two laws to establish proper safeguards for the property established by the so-called "spontaneous architecture" promoting, for instance, the recovery and the increase in value of the trabocchi (Regional Law of December 1994, n.93: "Arrangement for the recover and increase in value of the trabocchi of the Teatine coast"). But if on the one hand local institutions have confirmed their role as guarantors of the cultural property value represented by the "Genius loci", on the other they have not yet devoted all their resources to promote the image of this area with an advertising campaign that would present the "coast of trabocchi" as a "recognizable mark". The project of the shifting of the railway line constituted the further reason of the rediscovery and reutilization of this property. This is because, until now, the railway which ran along the waterline has preserved this portion of coast from the savage tourist colonization impeding the use of the coastal zone.

\section{The knowledge of trabocchi and of their environment}

It is necessary to know this heritage in order to take a census that will help to individuate any manufactured product in its context. However, the general knowledge of the construction is crucially important to identify all the significant documentation relevant to the realization of the interventions. For every overflow, "specific registry cards" were then set up, with the aim of starting" reflections founded on the destiny of the property.

Three specific objects are defined:

- the conservation of the "local" insertion identity; in this sense, the enrichment of the identification data with information of geological type (sea bottom) and climatic-environmental (currents and waves) turns out to 
be important in explaining the conditions in which buildings are placed with the aim of noticing the role in the breakdown determination and the corrective clause; for instance, to improve the foundation systems and choose the materials more appropriate to the solicitations of the sea climate; the conservation of the local constructive identity implied in the recurring geometries, the typologies and the dimensions; while registering information on the recurring dimensional data of the covered surface (with the relative orientation) and the configured data and typological (with a summary of the minimum and maximum dimensions of the manufactured products and the constituent spaces which are normally used to meet the demands of

Table 1: $\quad$ Survey schedule.

\begin{tabular}{|c|c|c|c|}
\hline $\begin{array}{c}\text { Progressive } \\
\text { number }\end{array}$ & \multicolumn{3}{|c|}{ Modelling of the sequence of trabocchi } \\
\hline \multicolumn{4}{|c|}{ Territorial collocation and address } \\
\hline $\begin{array}{l}\text { The place } \\
\text { "geographical } \\
\text { location } \\
\text { identification" }\end{array}$ & $\begin{array}{l}\text { Image of the } \\
\text { environmental } \\
\text { contest and } \\
\text { access to the } \\
\text { trabocco } \\
\text { [extract of the } \\
\text { Chart a 1:5000 } \\
\text { of the } \\
\text { surrounding } \\
\text { with evidence of } \\
\text { the road and } \\
\text { access } \\
\text { infrastructures] }\end{array}$ & $\begin{array}{l}\text { Geological support: } \\
\text { - } \quad \text { emergent sector } \\
\text { - } \text { submerged sector } \\
\text { Climatic conditions } \\
\text { - predominant winds } \\
\text { - prevalent current }\end{array}$ & $\begin{array}{l}\text { Schematic planimetry } \\
\text { with reference to the } \\
\text { orientation of the winds } \\
\text { and of the sea currents }\end{array}$ \\
\hline $\begin{array}{l}\text { The system } \\
\text { "constitutive- } \\
\text { dimensional } \\
\text { identification" }\end{array}$ & $\begin{array}{l}\text { Image of the } \\
\text { trabocco } \\
\text { [general } \\
\text { photograph of } \\
\text { the system] }\end{array}$ & $\begin{array}{l}\text { Installing typology } \\
\text { - pier/cliff } \\
\text { subsystems } \\
\text { - footbridge (distance } \\
\text { from the coast) } \\
\text { - fishing plan } \\
\text { (dimension and } \\
\text { height on the sea) } \\
\text { - fishing device } \\
\text { - stabilizing grillwork } \\
\text { - bathing hut } \\
\text { - equipment }\end{array}$ & $\begin{array}{l}\text { Schematic planimetry } \\
\text { with the general } \\
\text { dimensions of the } \\
\text { handmade product } \\
\text { (the measures are those } \\
\text { denounced for the } \\
\text { licence; then there are } \\
\text { those result of the } \\
\text { image from the satellite, } \\
\text { even if only indicative) }\end{array}$ \\
\hline $\begin{array}{l}\text { The memory } \\
\text { "cultural and } \\
\text { historical } \\
\text { identification" }\end{array}$ & \multicolumn{2}{|c|}{ Historical notices and chronicles. } & $\begin{aligned} & \text { Essential data: } \\
& \text { - } \text { epoch } \\
&- \text { constructor } \\
&- \text { owner } \\
&- \text { bounds } \\
&- \text { utilization of } \\
& \text { the L.R. n.93 } \\
& \text { of 14th } \\
& \text { December } \\
& 1994 \\
&\end{aligned}$ \\
\hline
\end{tabular}


environmental and technical matters) one aims at constituting a first data package, graphical-constituent, provided to show the first results on the validity/possibility for the recovery and reuse of the handworks;

- the conservation of "memory", at last, undoubtedly essential constitutes an essential element not only for the physical recovery of the handworks, but also for a sense of belonging to a place and a culture.

In short, the objective of the census was to provide more detailed information about the relative situation, number and state of the systems "trabocco" and simultaneously, also of the environmental context situation in which such manufactured products are located. Notably, if the result of this work leads to the maintenance of the environmental stability it will not be able to expand the tourist flow to the coastline. Specific and accurate data regarding not only the manufactured products but also significant built and natural features of the surrounding context must be recorded. Furthermore, it will be pointed out those artificial (manufactured products and infrastructures) and natural (beaches and cliffs) "emergencies" that could have a primary role in the proposal of a new model of tourist fruition of the system connected to the trabocchi. Trabocchi will furthermore be able to set up the reference archetype for the bathing facility design, figuratively appropriated to the place and eco-sustainable.

\section{From the recovery to the regulations for the new construction of trabocchi}

The rehabilitation and revaluation of Trabocco Turchinio (planning and works direction by architect M. Borrone), located at Punta del Turchinio in the municipality of San Vito Chietino were executed in about 13 months (works started in June 2004 and were over in July 2005) as a result of the violent seastorm of the first days of the year 2003, numerous and immense structural damages occurred, which compromised the function and stability of the building. This experience became the bench test of the various compatible assumptions and above all the moment for the preservation of the constructive practises that now result in alienation. The planning action was inspired by the "Paper of Amsterdam" (1975), in which the principle of the "integrated conservation" is established; it associates the two concepts of the conservation/restoration and of the attribution of an appropriate use. In fact, the sense of this intervention has been directed to the impediment of actions which could have altered the authenticity of the recent past of the "fishing machines", involving all the actors who were aware of the complex, as well as fragile, heritage on which to operate. This environmental awareness has placed firm limits on the overall project of restoration and redestination of the object. The restoration took into account the need to provide the Trabocco Turchinio, (the only one public property named by Gabriele D'Annunzio in Triumph of the Death), with a function compatible with its nature, also through the functional coming up of materials within the bounds of their endurance, coming from the neighbouring areas, a function which did not involve violent modifications. Hence the idea to convert this structure into a place for didactic, scientific and cultural activities, also because history shows 
that the survival of trabocchi is tightly connected to their utilization. An awareness linked to the expectation that the evocative capacity of suggestion of these places can represent also a potentially important tourist attraction as is shown by the cultural and scientific interest of such areas, inducing us to think how their conservation is not only a cultural duty, but it may be also understood as the economic presupposition for a rearrangement compatible with their survival. The phases of the restoration work were carried out making a systematic disassembling of the trabocco and, more accurately, determining all the categories of technical elements (composing the technological units and the categories of technological units). This comes from a reading of the subsystems constituting the manufactured product in object: the footbridge, the flat of fishing, the fishing device, the bathing hut. Since we did not work on an ex-novo building but based our intervention on the existing one and above all considering the characteristics of the location of both Turchinio and trabocco on the rock more generally, it was impossible to operate, intervening on each technological unit one by one, or in other words according to a pre-arranged program which was meant for operations on all parts of the same unity.

This condition has induced us to repair a portion of the system at a time, for in this way only few elements belong to various technological units. So it has been possible to reduce the difficulties linked to the necessity to work on the water. In fact, the work conducted in movement by the shore allowed a portion of the system "renewed" to function as a framework for acting on the adjacent portion till reaching the elements located closer and closer to the open sea (where water reaches a depth of about 6 metres). The execution of these operations, perhaps the only ones we could realize, implies the use of these procedures adopting the most ancient method practiced in traditional maintenance architecture, such as the utilization of special materials and elements. In this respect, it is useful to underline some important aspects and to make explicit some properties that cannot be inferred from the reading of the written schedules.

The first technical elements object of the intervention have been those composing the structures of foundation and of vertical elevation; in the works of balancing and replacement of these elements materials similar to the original ones have been used, except for the elements of collection (originally made with threaded bars and iron bolts), which for the first time have been made with threaded bars and steel bolts to avoid the formation of rust. Monitoring on the acting and on the state of the just finished structure has revealed a very important datum which has not been assumed a priori: because of the movements due to the sea and wind actions, the steel bolts slowly unscrew themselves making the whole building unstable. Therefore, in the structure of trabocchi the rust also has a fundamental role which consists in creating the right friction and the necessary cohesion in the nuts between the wooden elements. In the light of this observation, the steel bolts have been substituted with new iron bolts similar to the original ones, while monitoring the structure and screwing the bolts several times until the action of the sea has not given up a fairly regular degree of rusting of the nuts retaining their full functionality. The peculiar aspect described above concerns even more the links between the elements of the structure of vertical 
elevation and those of the structure of horizontal elevation, as well as the links between the longitudinal and transversal elements of the structure of horizontal elevation. In particular, as regards the trabocco Turchinio, no alteration was made in the typologies of the structures of vertical and horizontal elevation which have remained those traditional and more ancient (the vertical structure defined only by one pole- till the level of pounding of the footbridge- and by two strips of poles slightly inclined at $\mathrm{V}$ - with the function of supporting for the cables constituting the parapet - and the horizontal structure defined by crossing elements of connection between the vertical pole and the longitudinal girders) in contrast with the alternative technologies used in the course of the years (see the vertical supports made with two crossing elements instead of only one pole).

The objective of not modifying the characteristics of the structures trabocco led to an intervention which has respected as far as possible the materials and the traditional constructive techniques (in the structures of elevation, in the closing, in the partitions and in the functional equipment). With reference to this aspect, it is significant to point out the characteristic and "strategic" arrangement of the cables constituting the stabilizing system of connection between the antennae, the little antennae, the antenna poles and the poles of the hoist. The system of cables supporting the antennae and suspended to the antenna poles is fixed also to the hoist poles in a criss-cross way with respect to the fishing plan, that is the
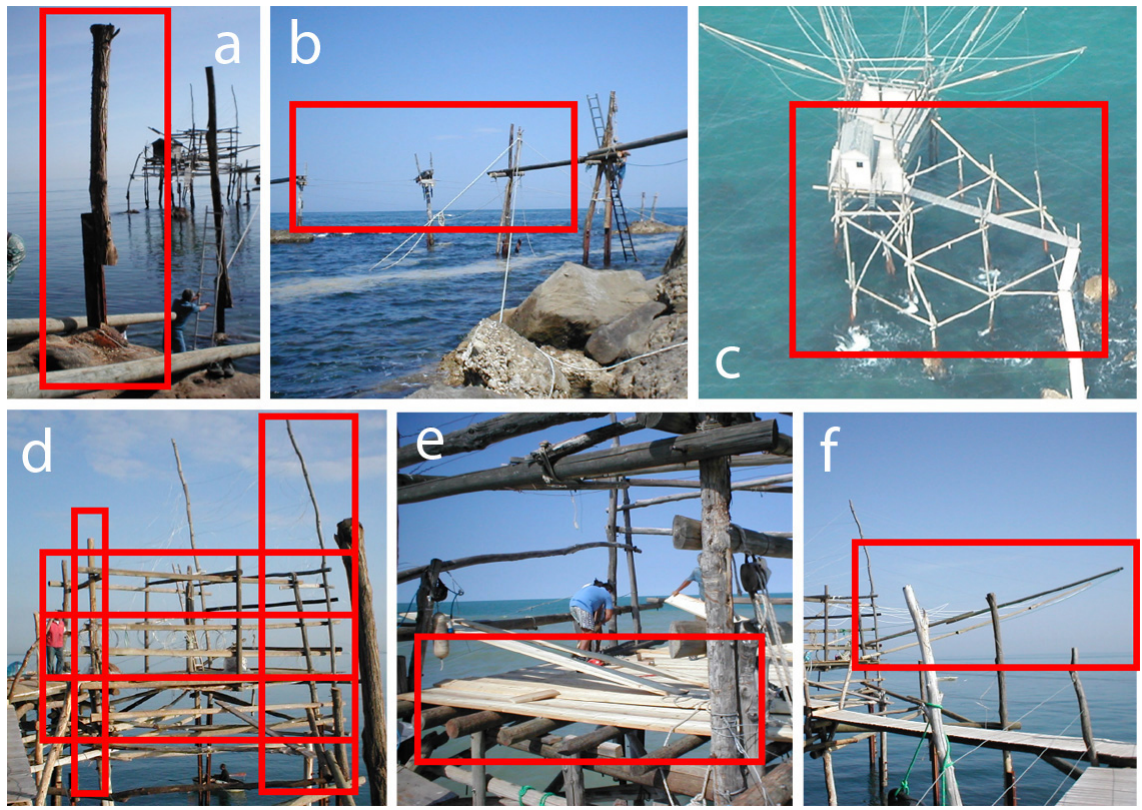

Figure 2: Images of the rehabilitation process: a) foundations and vertical elements of the footbridge; b) horizontal structure of the footbridge; c) stabilization structures; d) vertical and horizontal structures of the fishing plan; e) fishing plan partition elements; f) structures of the fishing device. 
tie-beams of the antenna and of the antenna pole on the right, connect to the pole of the hoist of left and vice versa for the left antenna. While the tension rods which support the little antennae are still attached to the antennae and hoist poles, but follow a different path: cables of the little left antenna connect before to the left antenna pole then to that of right and at last the hoist pole, always on the right (never crossing on the fishing plan); the same happens for the tension rods of the little right antenna. This tension rod arrangement defines a system of cables which, following various directions, stiffens the whole system supplying with constraints the vertical and horizontal displacements of the structural parts. This stiffening is implemented by the link elements between the antennae and the little antennae. The intervention made on the Trabocco Turchinio represents an important and useful experience to organize an appropriate, ordinary and continuative activity of maintenance and balancing of the trabocco. This intervention provides precious advices to complete the theoretical indications with precautions for practical applications.

\subsection{First conclusions about the construction of new trabocchi}

The most important "conclusions" drawn from the experience of the realization of this intervention (and which can be considered in any future works) are essentially related to the peculiarities of the kind of construction in object and to its installing conditions (in other words from the need to bring and assemble, following particular procedures, the constructive elements in the sea) and concern:

- reaching the shipyards;

- the problems of security in the shipyards;

- the peculiar constructive models;

- the peculiarities linked to the originality of each structure trabocco and its installing conditions.

The aspects mentioned above are to be considered not as problems to overcome but as bonds to respect. In this attempt to respect the environmental characteristics of the places, the way to the shipyards can at most be ameliorated (by cleaning the uncultivated vegetation, for instance) but not certainly make it easier (by twisting the hills sloping down the sea with roads). Moreover, the problems related to the security of workers and to the peculiar constructive models have to be tackled considering the characteristics of the situation without modifying the traditional technical solutions.

In short, in order to control by regulations the recovering of the trabocchi, we shall state our impossibility to generalize rules, as well as the necessity of leaving a border of flexibility for each case which depends on the originality of each single structure and on its installing conditions. The control system of the more important aspects of planning can foresee a comparison with various qualifying aspects as: morphological quality - the whole typological and morphological conditions of a building or installing organism which guarantee the safeguard and the increasing in value of the context and satisfying qualitative levels in terms of architectonic, relational and perceptive relations; eco-systemic 
quality - the systemic praxis to realize and guarantee conditions for wellbeing with respect to the pre-existing ecosystems along with a rational employment of the natural resources available; useful quality - the whole conditions which guarantee a suitable utilization of the building organism or of the installing complex by the consumers, with particular attention to the questions concerning the elimination and the overcoming of the architectonic barriers, the security of utilization and in general the needs of the new model of life referred to the weaker social users; system quality (process and product) - the organizing structure, responsibility, procedures, acting, techniques and operating activities put in action to satisfy the properties and the characteristics of a product which permit to respond to implicit or expressed needs.

The comparison between the project and the various performances can be disciplined by a control model of the single interventions synthesized in a project schedule where the following issues are scrutinized: the collocation suitable to the context, where the historic places of location and the distance between the different settlings are to be considered; the morphological and dimensional features of the manufactured product mentioned above, where the proportions of the subsystem (footbridge, fishing device and bathing hut) and the extensions of the relative spaces are to be considered; the definition of a suitable structural system, where the referring typology (in particular for the subsystem footbridge) and the geo-compatibility of the foundations are controlled; the choice of an appropriate constructive system, guaranteed from the responsible selection of materials and assembling techniques and also verified in its endurance, maintenance and functionality; the configuration of a complete organization of the spaces and of the surrounding environment, in order to evaluate the possibilities of fruition and adaptability of the spaces.

\section{The model of tourist fruition and defining sustainability indicators for equipment on the coast}

The promotion of good quality tourism, referred to a sustainable territory development in the specific case of the Theatine coast, needs to begin with the maintenance of the trabocco and the recovery of the constructive lesson of the "genius loci". It results from the recognition of the role these manufactured products could play on the level of landscape image as well as their function in suggesting conditions for areas specially equipped for bathing. In short, it is a question of remembering that the elements, especially the fragile ones, on which the promotion of the territory is based, risk to be penalized in a project which is not evaluated in all its implications. In these territories of extreme vulnerability, from the physical point of view and of their image, tourism can be configured as a risk for the environmental deterioration (intending for this not only the physical environment but also the social and economic one): the anthropological pressure, the congestion, can contribute to a sudden disqualification of the environment; the "savage" and inappropriate utilization of the territory, and also the construction of other trabocchi with inadequate technologies and materials can, in a short time, cancel the historic-cultural memory of the place, to give space to 
an homologate landscape or alienated by false icons of the local material culture. This is the case also for the isolated architectonic manufactured products and for the little and characteristic built-up areas which cover the coast; without an organic and common "plan", the interventions of both recovering and renewing, but more importantly the new constructions can alienate the peculiarity of the place and destroy those points of strength which today represent a possible alternative to the existing tourist offer, promoting eco-cultural activities. The increase in value of the system of naturalization, the model of bathing coast equipment, the relationship with the hinterland anthropological products (urban and agricultural), the recovery and the reuse of manufactured products emergent as reference in a service system, the proposal of an alternative and sustainable mobility system all are part of a unique, complex and integrated project where every action is strictly connected to the other ones and every choice must be evaluated with respect to the results in the other sectors. Choosing the trabocchi as "Manifesto" for the future development of the Theatine coastal territory means giving an objective where the choice evaluation and the balance between the options is the leading reason of the political and planning actions. There is no doubt that the knowledge of the trabocchi has been critical for an appropriate proposal for such a project, providing in parallel the guidelines for the construction of new buildings of the same kind. Moreover, it has made possible the identification of the elements necessary to the definition of a "surrounding" and to the increase in value. It will at last make it possible to make reflections on the sustainability indicator identification for the compatible equipment on the coast in object.

\subsection{Tutelage and increase in value of the natural property}

The coastal territory characterized by the presence of trabocchi shows environmental resources, and natural and anthropological-cultural property of remarkable interest. With the aim of promoting a sustainable tourist development, eco-cultural, we should put in evidence a few elements with a high power of attraction, that are central crux of the matter (functional to the definition of an organic arrangement of the entire area). The point of strength of these ambits is given by the character of the naturalization, still quite integral, that connects them; there are, however, territories not able to support this, for their scarcity on one side, and their geological consistency on the other, a solid tourist load. Hence, the weakness of a mass tourism characterized by residences on the coast and "heavy" and permanent bathing facilities. The naturalization, element of attraction, would be briefly destroyed with consequent decay of the tourist demands. The cataloguing of the specific ambits will contain the description of the "place" particularly for the safeguard of the environment in connection with the geological characteristics of the cliff and those hydrogeological of the waterline. The objective tends to guarantee the harmonization of each action-intervention with the characteristics of the place; the specific qualifications consist of the project of landscape integration. The indicator of control is given by the presence and the maintenance of the characteristics of the 
context to verify through the relief, ante and post intervention, and by the permanence of the perceptive peculiarities of the place itself.

\subsection{Recovery and reuse of the property of the old hill top villages by the coast and the agricultural industry}

In these territories the proposal of a sustainable development means an offer of various tourism segments such as historical, cultural, environmental, enogastronomic routes which are particularly useful for redistributing, in a more balanced way, functions and attractions. The same territory places itself with those elements suitable for supporting such offers and producing, therefore, a readjustment of the internal, often abandoned and underestimated areas, as regards the inhabited centres, and for the agricultural areas. The tourist request for residence, for instance, can be widely met with the presence of the heavy property in the old city centres and in the rural areas, as well as in the diffuse settlements; in such a way that there will be a more interesting offer which includes other attractive factors related to history and local culture, all the while respecting the new tourist building. The recovery and the reuse of big manufactured products which have been cast off, such as the railway stations, the workshops and other systems placed in strategic points on the territory, constitute further resources not to be alienated but to recover appropriately also considering in addition the role of land deterioration. The idea begins to take root as "a building constitutes a supply of invested natural capital, and that could be maintained during the time" (R. M. Pulselli, E. Tiezzi, Città fuori dal caos, la sostenibilità dei sistemi urbani, Donzelli Editore, Roma 2008. p.133) just through actions privileging interventions on the built products rather than on new ones and that, as consequence of it, introduce even the city and the building in a new cyclical process.

\subsection{New mobility}

For the bathing areas, the dismantling of the railway provides a "network" which would be able to determine a truly integrated bathing system of articulated served and serving spatial units as well as to unify the access to the trabocchi and the relevant and adjacent little beaches. The tunnel system utilization, once reinserted in the unity of the way, besides allowing the trial course of the whole path, will be able to supply a service system. The proposed mobility system will have to be verified on the base of energy systems put in action and of relating emissions.

\subsection{Conclusions: for a sustainable model of stretch of stand equipment}

The by now consolidated image of the trabocco, which is evocative of the Theatine coast, functions as the most appropriate "morpho-technological" pattern for this scenario, but also as a transferable model to other contexts characterized by similar physical-territorial connotations. In other words, the planning of the bathing facilities can adopt the lesson of trabocchi as a fundamental strategy and refer: 
- to the well-established image of the system as light, fairly good and "transparent", demountable and reconstructable manufactured product;

- to the installing typology, the pile-dwelling, as a system that "conquers" the sea where there is scarcity of earth, and consequently, to the "measured" dimensions and consistency with respect to the place characteristics and the vulnerability of the geological support. It comes out a planning attitude in which the interventions assume a shape in a way inversely proportional to the place vulnerability and make reference, for instance, to a system of ecoplanning based on a modular system able to be suited, (diversifying the offer and the elements, to the place demands);

- to appropriate technologies with the utilization of materials connoted from a high sustainability level (renewable origin, recycling easiness, in short subordinated to LCA/life cycle assessment) and with simple and feasible techniques, to a constructive and maintaining level, by a not skilled labour, above all in relation to the dismounting and seasonal remounting, matter of primary importance for the maintenance of the total environmental quality.

The specifications may define in particular the aspect of the manufactured products - which is shaped according to the parameters of sustainability - and privilege the reference to local material culture; it is preliminary, then, the study of the material culture and the recovery of the local constructive traditions. The specific objective is in this case the safeguard of the local constructive traditions. In short, it is requested a specific tutelage for the local material, constructive and technological characters. The strategies to act, at both planning and technological levels, refer to the adaptability of the manufactured products to the morphological characteristics of the sites; to the usage of local materials and techniques; to the reinterpretation of the constructive systems and to the morphological constructive techniques of less impact on the ground and more efficient from the energy and transports point of view. The control indicator used will consist in the verification of the presence or compatibility or comparison of the technical-constructive peculiarities and of the planning materials with the context in which the intervention is introduced. The necessary instruments will lead to the repertory evaluation of chosen materials and to the proposed techniques in relation to evaluations with LCA methodologies and of "ecological mark". Visual simulation of the intervention will be used for the evaluation of the correct installing.

\section{References}

[1] Forlani M.C., (2005). I trabocchi, archetipi costruttivi della leggerezza. Il Progetto Sostenibile. vol. 08, pp. 20-29 Edicom Edizioni

[2] Forlani M.C., (2005). El conocimiento de la tradicion constructiva local de los Abruzos (trabocchi, pinciaie, caciare) para una nueva sustentabilidad proyectual. (vol. I, pp. 395-406). in S. Huerta (a cargo di), Historia de la construccion, Actas del Cuarto Congreso Nacional, EFCA, Torrejon de Ardoz (Madrid). 
[3] Forlani M.C., (2005). Una proposta di attrezzature eco-compatibili per il turismo balneare. (vol. 1, pp. 175-183) in A. Passaro, a cura di, Attrezzature temporanee ecocompatibili per il turismo nelle aree costiere, Atti del convegno internazionale "Abitare verde", Luciano Editore, Napoli

[4] Forlani M.C., Radogna D. (2004). Trabocchi: a Sustainable Technology for Marine Environment. (vol. III, pp. 269-274) In $8^{\circ}$ World Conference on Timber Engineering. Proceedings of the WCTE 2004, Helsinki 